\title{
The Explicit Inverse of a Tridiagonal Matrix
}

\author{
By P. Schlegel
}

\begin{abstract}
The closed form inverse of a tridiagonal matrix, which is a slight generalization of a matrix considered by D. Kershaw (Math. Comp., v. 23, 1969, pp. 189-191), is given in this note. If the matrix has integer elements, an integer multiple of the inverse can be computed by integer arithmetic, that is, without machine roundoff error.
\end{abstract}

Forms of $B$ and $B^{-1}$. Let $B=\left(b_{i j}\right)$ be an $n \times n$ matrix given by

$$
\begin{aligned}
b_{i j} & =b_{i}, & & i=j, \\
& =\delta_{i, j-1}, & & i<j, \\
& =\delta_{i-1, j}, & & i>j,
\end{aligned}
$$

where $b_{i}=b_{n-i+1}$ and $\delta_{i j}$ is the Kronecker delta. Define $r_{k}=-\left(b_{k} r_{k-1}+r_{k-2}\right)$, $k=2, \cdots, n-1$, and $r=\left(b_{n} r_{n-1}+r_{n-2}\right)$, where $r_{0}=1$ and $r_{1}=-b_{1}$. Let $C=\left(c_{i j}\right)$ be an $n \times n$ matrix, where

$$
\begin{aligned}
c_{i j} & =r^{-1} r_{i-1} r_{n-j}, & & i \leqq j, \\
& =c_{i i}, & & i>j,
\end{aligned}
$$

then $C=B^{-1}$. An elementary but lengthy proof of this is to show that $B C$ is an $n \times n$ identity matrix. It can also be shown that $r=(-1)^{n+1} \operatorname{det}(B)$. This implies that $r_{i-1} r_{n-j}=(-1)^{n+1} A_{j i}$, where $A_{j i}$ is the cofactor of $b_{i i}$.

If $b_{i}, i=1, \cdots, n$, is an integer, then $r_{k}, k=2, \cdots, n-1$, and $r$ are integers. This implies that $r c_{i j}=r_{i-1} r_{n-i}$ is an integer, that is, an integer multiple of $B^{-1}$ can be generated by integer arithmetic. Similarly, if one had a linear system to solve, which was represented by a matrix of the form (1) with integer elements, then an integer multiple of the solution could be computed by integer arithmetic if the known vector had rational coordinates.

Generalization to Partitioned Matrices. If the elements $b_{i}, i=1, \cdots, n, 1,0$ of $B$ are replaced by $m \times m$ matrices $B_{i}, i=1, \cdots, n, I, 0$, where $I$ is the identity matrix and 0 is the null matrix, respectively, then the inverse of $B$ is given by the partitioned matrix (2) if $B_{i} B_{i}=B_{i} B_{i}, i, j=1, \cdots, n$. Here $r^{-1}$ is considered the inverse of some $m \times m$ matrix.

Applied Mathematics Division

ARDC, BRL

Aberdeen Proving Ground, Maryland 21005

Received November 26, 1969.

AMS Subject Classifications. Primary 1510, 1515; Secondary 1079, 1548.

Key Words and Phrases. Inverse, tridiagonal matrix, integer arithmetic, integer elements, partitioned matrices. 